\title{
Nomograms for predicting adverse obstetric outcome in IVF pregnancy: A preliminary study
}

\author{
Alice Portal MW ${ }^{1,2}$ | Claire Sunyach PhD, MW ${ }^{2}$ | Anderson Loundou PhD ${ }^{3}$ | \\ Odile Lacroix-Paulmye PhD, $\mathbf{M D}^{2}$ | Jeanne Perrin Pr, PhD, $\mathbf{M D}^{2,4}$ | \\ Blandline Courbiere Pr, PHD, MD $^{2,4}$
}

${ }^{1}$ Ecole Universitaire de Maïeutique de Marseille Méditerranée (EU3M), Aix Marseille Univ, Marseille, France

${ }^{2}$ Department of Gynecology-Obstetric and Reproductive Medicine, AP-HM, Hôpital La Conception, Marseille, France

${ }^{3}$ Public Heath Department, Aix-Marseille Université, Assistance Publique des Hôpitaux Marseille and Aix-Marseille Univ, Marseille, France

${ }^{4}$ CNRS, IRD, IMBE, Avignon Université, Aix Marseille Univ, Marseille, France

\section{Correspondence}

Blandine Courbiere, Department of Gynecology-Obstetric and Reproductive Medicine, AP-HM, Hôpital La Conception, 147 Bd Baille, 13005 Marseille, France. Email: blandine.courbiere@univ-amu.fr

\section{Funding information}

This study is part of the Investissements

d'Avenir program controlled by the National Research Agency (Projet CREER A*MIDEX; $\mathrm{n}^{\circ}$ ANR-11-IDEX-0001-02). CS is supported by ARS PACA (Regional Agency for Health, Provence-Alpes-Cote d'Azur C2017-000265) and Region Sud Provence-Alpes-Cote d'Azur (C20171348) through funding of the Plateforme CREER (Couple Reproduction Enfant Environnement et Risque).

\begin{abstract}
Background: In a previous study, we showed that the obstetric complication rate after in vitro fertilization (IVF) pregnancy was $40 \%$. The main objective of our study was to determine maternal prognosis factors that influence the IVF pregnancy outcome.
\end{abstract}

Methods: We conducted an observational retrospective monocentric study between January 2014 and January 2018. Pregnancy over 22 gestational weeks (GW) obtained after IVF in our infertility clinic was included. Maternal characteristics and pregnancy outcome were collected.

Results: Data from 498 IVF pregnancies were analyzed. The most significant maternal prognosis factors for obstetric complications were maternal age above 40 years (OR 3,0 [95\% IC 1,30-7,09], $P=0,010)$, twin pregnancies (3.8 [95\% IC 1.49-9.99], $P=.005)$, daily maternal smoking above 10 cigarettes (7.1 [95\% IC 1.22-41.74], $P=.029)$, maternal obesity (2.2 [95\% IC 1.19-4.07], $P=.012)$, endometriosis stages III and IV (6.4 [95\% IC 1.52-27.04], $P=.011)$, and history of ovarian hyperstimulation syndrome (OHSS) in early pregnancy (5.7 [95\% IC 1.29-24.74], $P=.021$ ). Risk increase was independent of pregnancy type (singleton or twin) and allowed the elaboration of 2 nomograms.

Conclusions: Our study showed a link between some maternal factors and increase in obstetric complications after IVF. Screening of these factors during preconceptional visit is essential to identify at high-risk pregnancies and adapt their monitoring.

\section{K E Y W O R D S}

IVF, nomogram, pregnancy outcome

\section{INTRODUCTION}

In France, one out of seven couples consults for infertility and one out of ten requires assisted reproductive treatments (ART). ${ }^{1}$ In vitro fertilization (IVF) has become a standard

Alice and Claire authors contributed equally to the study. method for most types of fertility problems. According to estimates, each year, 3\% of French newborns are conceived after in vitro fertilization (IVF). It has been observed that both infertility and IVF are associated with compromised maternal and infant perinatal outcomes. After ART, pregnant women have an higher risk to experience pregnancy-related complications (ie, high blood pressure, gestational diabetes, 
placenta previa, placental abruption, metrorrhagia, postpartum hemorrhage, hydramnios, small for gestational age (SGA), premature birth, and cesarean) compared with those who conceive naturally. ${ }^{2-6}$ In our ART center, we previously showed that obstetric complication rates after IVF were $40 \%$ and that $31 \%$ of IVF pregnancies required hospitalization. ${ }^{7}$ Pregnancies that result from fresh or frozen embryos may contribute to different obstetric and neonatal complications. ${ }^{8-11}$ In a large meta-analysis, in singleton pregnancies conceived from thawed embryos, Maheshwari and colleagues reported lower risks of preterm birth and SGA, but higher risks for hypertensive disorders of pregnancy and high birthweight compared with IVF pregnancies after fresh embryo transfers. ${ }^{9}$ The impact of embryo culture before blastocyst-stage embryo transfer is still debated. However, in a study analyzing 67147 IVF cycles, no increased risks of preterm birth nor low/high birthweight after fresh blastocyst-stage embryo transfer $v s$ cleavage-stage embryo transfer were found. ${ }^{12}$

Predicting adverse obstetric outcomes in ART pregnancies is therefore an important issue for counseling pregnant women and personalizing the pregnancy follow-up. With the aim of helping counseling and management of these pregnancies, we conducted a pilot study for studying maternal risk factors of obstetric complications to generate nomograms for screening higher risk IVF pregnancies.

\section{2 | METHODS}

\section{1 | Patients}

From January 1, 2014, to January 31, 2018, women pregnant after IVF or ICSI-IVF treatment at the ART Center of the University Hospital were retrospectively identified. All of them had been unfertile for at least one year. Both pregnancies after fresh and frozen-thawed embryo transfer have been considered. Patient's characteristics were as follows: maternal age, BMI, tobacco consumption, and AMH rate. Obesity was defined as a BMI $>30 \mathrm{~kg} / \mathrm{m}^{2}$. The infertility characteristics recorded were as follows: gravidity, parity, the duration of infertility before pregnancy, and the etiology of infertility (oligo-astheno-teratospermia (OATS), tubal obstruction, endometriosis, and polycystic ovary syndrome (PCOS)). We retrieved from medical records the severity of endometriosis that was scored by physicians according to the rAFS Classification. Some characteristics of the ART cycle that led to live birth were also collected: number of IVF / ICSI attempts before pregnancy, fresh or frozen-thawed embryo transfer, early or late ovarian hyperstimulation syndrome (OHSS), and bleeding during first trimester. Pregnancy outcomes were as follows: singleton or twin pregnancies and bleeding (gestational diabetes mellitus, intrauterine growth restriction, preterm rupture of membranes and preterm labor, pregnancy-induced hypertension $(\mathrm{PIH})$ antepartum hemorrhage, postpartum hemorrhage, and preeclampsia) using international definitions and vaginal delivery or cesarean.

\section{2 | Procedure}

Each year in France, medical and scientific data from ART centers throughout the country are reported to the Biomedicine Agency under the control of the French Health Ministry. These data were used for this study. Before undergoing IVF, patients were consented or not for the possibility to analyze retrospectively their medical data. Patient decision was recorded in their medical file. The study was approved by the local ethics committee of our university.

Descriptive maternal characteristics and pregnancy outcomes were collected from patient's medical records and anonymized. We excluded pregnancies after oocyte or sperm donation.

Any pregnancy associated with at least one of the following obstetric or fetal complications was considered as pathological: prepartum hemorrhage, high blood pressure, gestational diabetes, obstetric cholestasis, placenta previa, placental abruption, preterm rupture of membranes, preterm labor, intrauterine growth restriction (IGHR), vanishing twin's syndrome, preterm birth, and postpartum hemorrhage.

\section{3 | Statistical analysis}

Statistical analysis was performed using the SPSS software (SPSS, Inc, Chicago, IL). The end point of the study was obstetric complications after IVF and ICSI-IVF pregnancies. Variables entered into the model were as follows: patients and infertility characteristics, pregnancy outcomes, and all the obstetric complications mentioned above. The chi-square and the Student $t$ tests were performed. Multivariable logistic regression analysis was used to test the association between obstetric complications and maternal characteristics. The odds ratio is presented with their $95 \%$ confidence interval (95\% CI).

Backward variable selection was performed to determine independent covariates. We decided to test the 4 obstetric complications with the highest incidence in our population, that is, gestational diabetes mellitus, intrauterine growth restriction, preterm rupture of membranes, and preterm labor.

Multiple logistic regression was used to build two nomograms. Cofactors were selected for further inclusion in the nomogram in the case of univariate $p$ values $\leq 0.2$. The first nomogram predicts obstetric outcomes in all the pregnancies obtained after IVF and ICSI-IVF and the second predicts these obstetric outcomes only in the singleton pregnancies. They allowed a visual assessment of 
each predictor variable significance in the obstetric complications' probability. A $p$-value $<0.05$ was considered significant.

\section{3 | RESULTS}

\section{1 | Population characteristics}

A total of 516 pregnancies with a delivery after 22 gestational weeks were recruited in the ART center of the University hospital. We lost contact with 11 patients, and 7 had incomplete medical record. A total of 498 pregnancies were included in the study.

The mean women age was $32.1 \pm 4.4$ years. They had been infertile for $4.4 \pm 2.6$ years before starting ART and had $2 \pm 1$ IVF, ICSI-IVF, and frozen embryo transfer (FET) cycle attempt. The mean body mass index (BMI) was $24.4 \pm 5.2 \mathrm{~kg} /$ $\mathrm{m}^{2}$ with $17 \%$ overweight $(\mathrm{n}=85)$ and $15 \%$ obese $(\mathrm{n}=72)$ women. In our population, $30 \%$ women smoked $(n=143)$, among whom $13 \%$ smoked more than 10 cigarettes a day $(\mathrm{n}=64)$.

Infertility etiologies were as follows: tubal obstruction $(26 \%, \mathrm{n}=126)$, endometriosis $(13 \%, \mathrm{n}=66)$ of which $8.2 \%$ $(\mathrm{n}=41)$ were of stages III-IV, polycystic ovary syndrome $(10 \%, \mathrm{n}=50)$, and male infertility $(38 \%, \mathrm{n}=186)$. Early and late ovarian hyperstimulation syndromes were observed in 16.5 percent $(\mathrm{n}=82)$ of the pregnant women assessed.

In our study population, $41.1 \%$ of the pregnancies have been obtained through IVF-fresh embryo transfer $(n=204)$, $37.7 \%$ through ICSI-fresh embryo transfer $(\mathrm{n}=188)$, and $21.1 \%$ through FET $(\mathrm{n}=105)$. The rate of fresh and frozen embryo transfers was $78 \%(\mathrm{n}=389)$ and $22 \%(\mathrm{n}=109)$, respectively. Among all pregnancies, $79 \%$ were singleton $(\mathrm{n}=394)$ and $21 \%$ were twins $(\mathrm{n}=104)$. Obstetric complication rate was $38.7 \%(n=193)$, and the most frequent adverse outcomes were as follows: preterm labor $(14.8 \%$, $n=74)$, gestational diabetes mellitus $(8.4 \%, n=42)$, intrauterine growth restriction $(7.2 \%, \mathrm{n}=36)$, preterm rupture of membranes $(6 \%, \mathrm{n}=30)$, and hypertensive disorders $(4.4 \%$, $\mathrm{n}=22)$ There were more vaginal deliveries $(63.4 \%, \mathrm{n}=316)$ than cesarean $(36.5 \%, \mathrm{n}=182)$.

\section{2 | General Obstetric complications}

Patient's characteristics linked to adverse pregnancy outcomes are summarized in Table 1. Obesity (BMI $>30 \mathrm{~kg} /$ $\mathrm{m}^{2}$ ), active smoking $>10$ cigarettes/ per day during ART, severe endometriosis, and twins were independent risk factors associated with a significant increased rate of pathological pregnancies. Our results were not significant for advanced maternal age above 40 years ( $p$-value 0.2 ).
Twin pregnancy was the major factor responsible for obstetric complications with an increased risk of 3.8 (OR 3.8 [95\% CI 1.49-9.99], $P=.005$ ). For singletons, advanced maternal age above 40 years and maternal obesity were significant risk factors for adverse pregnancy outcome, with a threefold increase (OR 3.0 [95\% CI 1.30-7.09], $P=.010$ ) and a 2.2-fold increase (OR 2.2 [95\% CI 1.194.07], $P=.012$ ), respectively. Gestational diabetes risk increased by 1.5 (OR 1.5 [95\% CI 1.01-2.32], $P=.048$ ) with history of failed ICSI-IVF cycle. Daily maternal smoking above 10 cigarettes increased the risk of preterm rupture of membranes by 7 (OR 7.1 [95\% CI 1.22-41.74], $P=.029$ ) compared with nonsmoking women and women smoking less than 10 cigarettes a day. Risk of preterm labor was significantly increased by 4 factors: high number of IVF cycles (OR 1.6 [95\% CI 1.05-2.41], $P=.029$ ), daily maternal smoking above 10 cigarettes (OR 3.8 [95\% CI 1.31-10.72], $P=.013$ ), uterine malformation (OR 5.0 [95\% CI 1.2919.33], $P=.020$ ), and endometriosis stages III-IV (OR 6.4 [95\% CI 1.52-27.04], $P=.011)$.

Maternal smoking status (OR 8.2 [95\% CI 1.64-40.88], $P=.010)$ and ovarian hyperstimulation syndrome (OR 5.7 [95\% CI 1.29-24.74], $P=.021$ ) increased intrauterine growth restriction (IUGR) risk. Factors responsible for obstetric complication subgroup of our population of study were as follows: singleton pregnancy, twin pregnancies, pregnancies with gestational diabetes mellitus, pregnancies with preterm rupture of membranes, pregnancies with preterm labor, and

TA B LE 1 Patient's characteristics associated with increased rate of pathological pregnancies in univariate analysis

\begin{tabular}{|c|c|c|c|}
\hline & Parameters & $\begin{array}{l}\text { Pathological } \\
\text { pregnancies }\end{array}$ & $P$-value \\
\hline \multirow[t]{2}{*}{ Maternal age } & $\begin{array}{l}<40 \text { years old } \\
(\mathrm{n}=470)\end{array}$ & $38.1 \%(\mathrm{n}=179)$ & \multirow[t]{2}{*}{0.200} \\
\hline & $\begin{array}{l}>40 \text { years old } \\
(n=28)\end{array}$ & $50 \%(\mathrm{n}=14)$ & \\
\hline \multirow[t]{2}{*}{ Obesity } & $\begin{array}{l}\mathrm{BMI}<30 \\
(\mathrm{n}=392)\end{array}$ & $35.7 \%(\mathrm{n}=140)$ & \multirow[t]{2}{*}{0.022} \\
\hline & $\mathrm{BMI}>30(\mathrm{n}=72)$ & $50 \%(\mathrm{n}=36)$ & \\
\hline \multirow[t]{2}{*}{$\begin{array}{l}\text { Maternal } \\
\text { smoking }\end{array}$} & $\begin{array}{l}<10 \text { cigarettes/day } \\
(\mathrm{n}=79)\end{array}$ & $30.4 \%(n=24)$ & \multirow[t]{2}{*}{0.043} \\
\hline & $\begin{array}{l}>10 \text { cigarettes/day } \\
(\mathrm{n}=64)\end{array}$ & $46.8 \%(n=30)$ & \\
\hline \multirow[t]{3}{*}{ Endometriosis } & Absence $(n=426)$ & $36.8 \%(\mathrm{n}=157)$ & \multirow[t]{3}{*}{0.038} \\
\hline & Stage III $(\mathrm{n}=16)$ & $62.5 \%(n=10)$ & \\
\hline & Stage IV $(n=25)$ & $56 \%(\mathrm{n}=14)$ & \\
\hline \multirow{2}{*}{$\begin{array}{l}\text { Pregnancy } \\
\text { type }\end{array}$} & Singleton $(n=394)$ & $31.5 \%(\mathrm{n}=124)$ & \multirow[t]{2}{*}{0.001} \\
\hline & Twin $(n=104)$ & $66.3 \%(\mathrm{n}=69)$ & \\
\hline \multirow{2}{*}{$\begin{array}{l}\text { Embryo } \\
\text { transfer }\end{array}$} & Single $(\mathrm{n}=148)$ & $29.7 \%(n=44)$ & \multirow[t]{2}{*}{0.007} \\
\hline & Double $(\mathrm{n}=350)$ & $42.6 \%(n=149)$ & \\
\hline
\end{tabular}


pregnancies with IUGR (Table 2). No case of stillbirth or neonatal death was recorded in our studied population.

Univariate analysis was used to determine factors responsible for pathological pregnancies, and multivariate analysis was used to determine clinically significant factors. Our analysis of 498 infertile women resulted in an original model to predict obstetric complications for each pregnancy based on clinically significant data. Covariates considered of our model included patient age, maternal smoking status, maternal obesity, endometriosis stages III-IV, ovarian hyperstimulation syndrome, and twin pregnancies. Independently of the pregnancy type (singleton vs twin pregnancy), several maternal prognosis factors affected IVF pregnancy adverse outcome. Two nomograms were developed considering covariates $(i)$ in the total pregnancies population and (ii) only in singleton pregnancies. The first nomogram (Figure 1) was developed in a cohort including all 498 pregnancies. The second nomogram (Figure 2) was developed in a cohort of 394 women with singleton pregnancies to eliminate the bias of multiple pregnancies.

\section{4 | DISCUSSION}

IVF and maternal factors relating to infertility and infertility treatments are associated with adverse pregnancy outcomes and infant perinatal outcomes. ART pregnancies must therefore be managed as potential high-risk pregnancies and must be monitored accordingly. Although preliminary, our study provides first nomograms to help clinician in the monitoring and follow-up of IVF pregnancy.
In France, National Perinatal Surveys (ENP) ${ }^{13}$ periodically present data on the main indicators of mothers' and newborns' health and risks factors, and medical practices during pregnancy and childbirth. In the present study, analysis indicated an increase in obstetric complication rate, with $39 \%$ pathological pregnancies compared with the $18.1 \%$ reported in the general French population reported in the 2016 ENP survey. Patient's clinical characteristics, that is, weight and smoking status, were similar in general population and in our study (Table 3). These results indicate that despite the monocentric design of our study, our population seems to be representative of the French population. We observed that the frequency of twin pregnancies and cesarean rates were higher ( $20.8 \%$ vs $1.8 \%$ and $36 \%$ vs $20.2 \%$, respectively) in our population than in the French population. This result is consistent with other studies. It is estimated that $19 \%$ and $21 \%$ of twins are because of IVF and other infertility treatments as ovulation induction. ${ }^{14}$

Twin pregnancies are well known to be the first cause of obstetric complications, as preterm birth, anemia, and cesarean. ${ }^{15}$ The high rate of twins observed after ART is because of the widely used double embryo transfer, before the development of a systematic strategy of single embryo transfer. ${ }^{16}$ At the time of our retrospective cohort, embryologists did not offer systematically a blastocyst SET, explaining the high incidence of preterm labor and preterm birth in our study.

In the present study, twin pregnancy was the most significant factor responsible for obstetric complications after ART, increasing this risk by fourfold. Accordingly, Paloma et al ${ }^{17}$ have shown that twin pregnancies after ART have a higher risks of preterm birth, preterm rupture of membranes,

TA B LE 2 Significant risk factors for pregnancies after ART in each population category in univariate and multivariable analyses

\begin{tabular}{|c|c|c|c|c|c|}
\hline & Risk factors & $\begin{array}{l}\text { Univariate analysis } \\
P \text {-value }\end{array}$ & OR & IC & $\begin{array}{l}\text { Multivariate analysis } \\
P \text {-value }\end{array}$ \\
\hline Singleton pregnancies & Maternal age $>40$ & 0.026 & 3,0 & [1.30-7.09] & 0.010 \\
\hline Twin pregnancies & Twin birth & 0.001 & 3.8 & [1.49-9.99] & 0.005 \\
\hline $\begin{array}{l}\text { Gestational diabetes } \\
\text { mellitus }\end{array}$ & $\begin{array}{l}\text { High rank of ICSI-IVF } \\
\text { cycles }\end{array}$ & 0,021 & 1,5 & {$[1.01-2.32]$} & 0.048 \\
\hline $\begin{array}{l}\text { Preterm rupture of } \\
\text { membranes }\end{array}$ & $\begin{array}{l}\text { Maternal smoking }>10 \\
\text { cigarettes/day }\end{array}$ & 0.197 & 7.1 & [1.22-41.74] & 0.029 \\
\hline \multirow[t]{2}{*}{ Preterm labor } & $\begin{array}{l}\text { High rank of IVF } \\
\text { cycles }>3\end{array}$ & 0.044 & 1.6 & {$[1.05-2.41]$} & 0.029 \\
\hline & $\begin{array}{l}\text { Maternal smoking }>10 \\
\text { cigarettes/day }\end{array}$ & 0.032 & 3.8 & {$[1.31-10.72]$} & 0.013 \\
\hline \multirow{2}{*}{$\begin{array}{l}\text { Intrauterine growth } \\
\text { restriction }\end{array}$} & Maternal smoking & 0.168 & 8.2 & [1.64-40.88] & 0.010 \\
\hline & $\begin{array}{l}\text { Ovarian hyperstimulation } \\
\text { syndrome }\end{array}$ & 0.023 & 5.7 & [1.29-24.74] & 0.021 \\
\hline
\end{tabular}


preeclampsia, and gestational diabetes mellitus. Similarly in their meta-analysis, Quin and colleagues ${ }^{4,5}$ reported higher rates of placenta previa, elective cesarean, preterm birth, very preterm birth, low birthweight, and congenital malformations in dichorionic twin pregnancies after ART.

However, and interestingly, when singleton pregnancies were only considered, rates of preterm labor and preterm birth remained higher than in the general French population, along with higher risk of cesarean. Our results are consistent with another study on a large cohort of births in France. ${ }^{18}$ Hence, and at the risk of stating the obvious, the first and easiest factor to change to reduce obstetric complications arising from IVF is to avoid twin pregnancy. Altogether, our results strongly advocate for a single embryo transfer policy after embryo culture as previously stated. ${ }^{16}$

Among the prognostic factors of adverse obstetric outcome, several factors were found independently of the type of pregnancy and allowed us to establish a first nomogram considering all pregnancies and a second nomogram for singleton pregnancies alone. These factors included the following: maternal age above 40, smoking during ART (10 cigarette per day), BMI above $30 \mathrm{~kg} / \mathrm{m}^{2}$, stage III and IV endometriosis, OHSS, and twin pregnancy. It is noteworthy that advanced maternal age is linked with an increased obstetric complication risk. ${ }^{19-21}$ In our study, the subgroup of women $>40$ years old was small $(n=14)$ and thus lacked statistical power. Paloma et $a l^{17}$ have shown that advanced maternal age above 40 years was linked with high-pressure blood, preeclampsia, gestational diabetes mellitus, preterm birth, cesarean, and an increased perinatal mortality. In singleton pregnancies, we found a threefold obstetric complication risk indicating that advanced maternal age was the most significant factor responsible for pathological pregnancies.

Daily maternal smoking above 10 cigarettes increased by 7 the risk of preterm rupture of membranes. Regardless of the number cigarettes smoked per day, maternal smoking was also linked to increased risk of preterm labor risk and fetal growth restriction. These data are in full agreement with the Waylen meta-analysis ${ }^{22}$ and Tons et al, ${ }^{23}$ which reported an increased risk of preterm birth and small gestational age after maternal smoking.

Together with the trend in delaying childbearing, the prevalence of overweight and obesity is also increased. Several studies have shown a more adverse effect of obesity among younger women undergoing IVF treatment ${ }^{2,24}$ and have shown the maternal obesity impact on miscarriages and perinatal complications after IVF. We showed that obesity increased by 2 the risk of obstetric complications.

Impact of endometriosis on pregnancy is currently controversial. Two studies have reported a higher risk of placenta previa, preterm birth, cesarean, and postpartum hemorrhage associated with endometriosis. ${ }^{25,26}$ In our study,
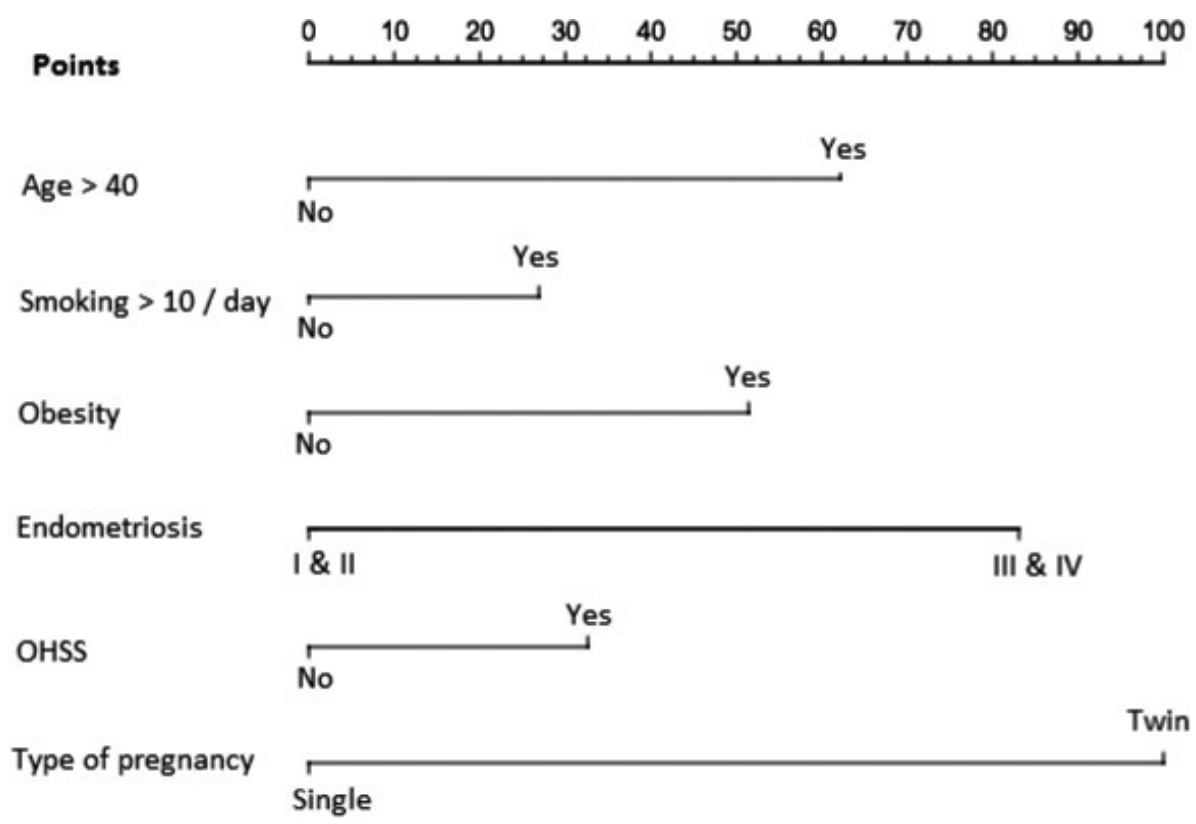

Total points

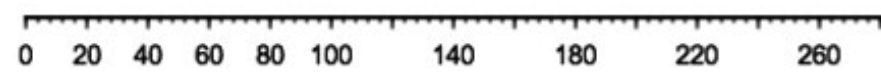

Probability

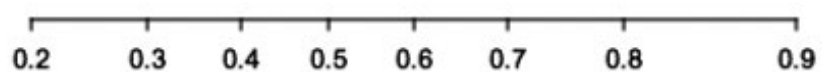

F I G U RE 1 Nomogram predicting obstetric complications for all pregnancies in the study $(\mathrm{n}=498)$ including 6 prognosis factors: maternal age above 40 years, maternal obesity, endometriosis stages III-IV, ovarian hyperstimulation syndrome (OHSS), and type of pregnancy 


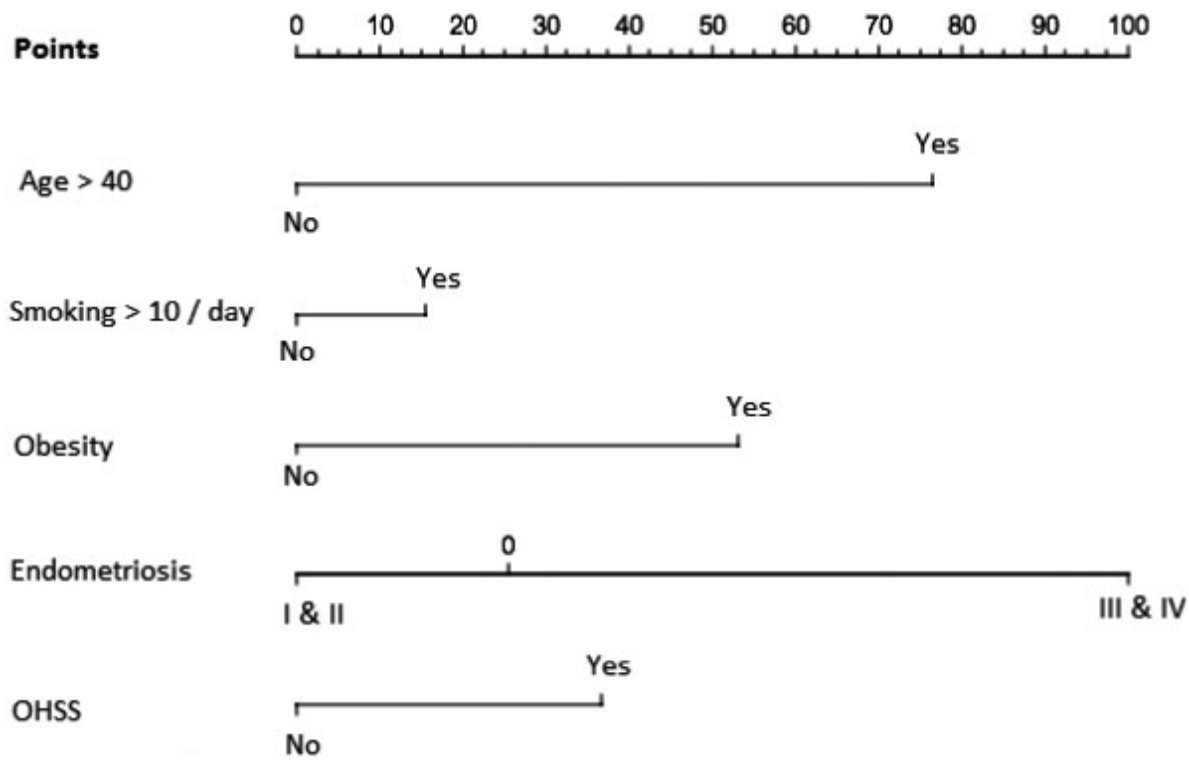

Total points

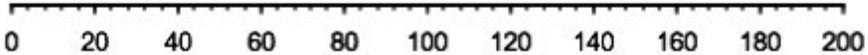

Probability

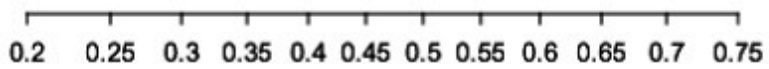

FI G URE 2 Nomogram predicting obstetric complications for singleton pregnancies in the study ( $\mathrm{n}=394)$ including five prognosis factors: maternal age above 40, maternal obesity, endometriosis stages III-IV, and ovarian hyperstimulation syndrome

T A B L E 3 Patients' characteristics of the study population compared with general French population ${ }^{13}$

\begin{tabular}{|lll|}
\hline Patients characteristics & $\begin{array}{l}\text { Population of the study } \\
(\mathbf{n}=\mathbf{4 9 8})\end{array}$ & $\begin{array}{l}\text { General population } \\
(\mathbf{n}=\mathbf{1 3} 384)\end{array}$ \\
\hline $\begin{array}{l}\text { Maternal smoking status } \\
\begin{array}{l}\text { Daily maternal smoking higher than } \\
10 \text { cigarettes }\end{array}\end{array}$ & \begin{tabular}{l}
$28.7 \%(\mathrm{n}=143)$ \\
\hline Overweight
\end{tabular} & $\begin{array}{l}12.4 \%(\mathrm{n}=62) \\
(\mathrm{n}=4015)\end{array}$ \\
\hline Obesity & $17 \%(\mathrm{n}=85)$ & $20 \%(\mathrm{n}=2677)$ \\
\hline Gestational diabetes mellitus & $14.4 \%(\mathrm{n}=72)$ & $11.9 \%(\mathrm{n}=1606)$ \\
\hline Intrauterine growth restriction & $8.4 \%(\mathrm{n}=42)$ & $7.2 \%(\mathrm{n}=964)$ \\
\hline Preterm labor & $7.2 \%(\mathrm{n}=36)$ & $5.4 \%(\mathrm{n}=723)$ \\
\hline Preterm birth & $14.8 \%(\mathrm{n}=74)$ & $5.4 \%(\mathrm{n}=723)$ \\
\hline Twin pregnancy & $17.6 \%(\mathrm{n}=88)$ & $7.5 \%(\mathrm{n}=1004)$ \\
\hline Cesarean & $20.8 \%(\mathrm{n}=104)$ & $1.8 \%(\mathrm{n}=234)$ \\
\hline Pathological pregnancy & $36.3 \%(\mathrm{n}=181)$ & $20.2 \%(\mathrm{n}=2704)$ \\
\hline
\end{tabular}

endometriosis stages III-IV increased the risk of obstetric complications, in particular preterm labor.

In addition, we have shown a link between ovarian hyperstimulation syndrome (OHSS) and the risk of intrauterine growth restriction. Our results align with previous works published both in our IVF center ${ }^{27}$ and in others, which showed that OHSS can increase the risk of thromboembolic complications and the risk of pregnancy-induced hypertension $(\mathrm{PIH})$ in IVF pregnancies. In this retrospective study, we used a long agonist protocol for patients at high risk of
OHSS. Since this study and following the literature, we have changed our controlled stimulation strategy, and we now prescribe antagonist protocol, agonist LH-RH triggering, and all-embryo freezing for all high-risk OHSS women. ${ }^{28}$

Some prognosis factors were not considered in our nomograms because of a lack of statistical power:

We observed that ICSI was a protective prognosis factor compared with IVF, and FET had no influence on obstetric complication rates. In our ART center, ICSI is offered only for severe sperm abnormalities, with mainly fertile women. 
This can explain the low rate of obstetric complications in "none-infertile" women. Infertility is a risk factor of obstetric complications per se, even in spontaneous pregnancies. Jaques et $\mathrm{al}^{29}$ have shown that infertile women having spontaneous pregnancy are more prone to develop PIH, preeclampsia, antepartum hemorrhage, preterm birth, and cesarean. Consistent with earlier studies, single or double embryo transfer was a significant risk factor of adverse outcome with $43 \%$ ( $n=149$ ) pathological pregnancy rate in double embryo transfer vs $30 \%(n=44)$ after single embryo transfer. Paloma et $\mathrm{al}^{17}$ have shown that double embryo transfer increased preterm birth risk. Double embryo transfer often induces twin pregnancy. Finally, we observed that a history of a high number of IVF cycles or of ICSI-IVF was associated respectively with higher risks of preterm labor and gestational diabetes mellitus. To our knowledge, this observation has never been reported in the literature, but needs to be further evaluated.

\section{1 $\quad$ Strength and limitation}

We are aware of the biases and limitations of our preliminary study. One limitation is the small size of our sample, which included singletons and twins, and fresh and frozen embryo transfers, giving rise to a lack of statistical power to analyze subgroups. Since fresh embryo transfer and frozen embryo transfer lead to different obstetric complication risks, ${ }^{8-11}$ we cannot rule out biases. This must be emphasized since in a meta-analysis Sha et al ${ }^{30}$ demonstrated that different IVF pregnancy-related complications result from transfer of cryopreserved versus fresh embryos. The nomograms that we derived from our results could nevertheless be useful for managing pregnancies in the context of assisted reproduction technologies. A national cohort study could allow construction of new nomograms based on more robust statistical analysis. Second, we did not analyze socio-economic level nor occupational exposures that both are well-recognized factors affecting pregnancy outcome. In addition, a threshold of 10 cigarettes/day was dictated by our clinical practice. We believe that above 10 cigarettes per day, it can be more difficult to quit. These women tend therefore to keep smoking during their pregnancy. In this subpopulation, smoking could account for increased risk of intrauterine growth restriction. Finally, and even if our sample seems representative of the French population when compared with the ENP data, we cannot completely rule out recruitment bias because of the retrospective and monocentric design of our study.

\section{2 | CONCLUSION}

Our monocentric study highlights that pregnancies achieved through assisted reproduction technology for infertile women are more exposed to adverse obstetric outcomes. Obstetric complications are because of maternal factors and the assisted reproduction technology itself, even in single pregnancies. Our work showed that medical visits and strict pregnancy counseling services are required for screening maternal risk factors (maternal age, smoked status, maternal obesity, endometriosis, and OHSS history, twin pregnancy). We believe that our nomograms are simple and useful tools to screen and adapt the follow-up of ART pregnancy. However, further prospective study to validate this new tool is warranted. Our study emphasizes the importance to act on modifiable risk factors such as obesity and smoking status. Our nomogram could be shown to couples during medical visit before starting infertility treatments to prompt early pre-ART smoking weaning and obesity reduction interventions.

\section{CONFLICT OF INTEREST}

The authors have no conflict of interest to declare.

\section{DATA AVAILABILITY STATEMENT}

The data that support the findings of this study are available from the corresponding author upon reasonable request.

\section{REFERENCES}

1. Agence de la biomédecine - rapport médical et scientifique. https://www.agence-biomedecine.fr/annexes/bilan2016/donnees/ procreation/01-amp/synthese.htm - Recherche Google. Accessed March 23, 2020

2. Luke B. Pregnancy and birth outcomes in couples with infertility with and without assisted reproductive technology: with an emphasis on US population-based studies. Am J Obstet Gynecol. 2017;217(3):270-281. https://doi.org/10.1016/j.ajog.2017.03.012

3. Szymusik I, Kosinska-Kaczynska K, Krowicka M, Sep M, Marianowski P, Wielgos M. Perinatal outcome of in vitro fertilization singletons - 10 years' experience of one center. Arch Med Sci. 2019;15(3):666-672. https://doi.org/10.5114/aoms.2019.82670

4. Qin JB, Wang H, Sheng X, Xie Q, Gao S. Assisted reproductive technology and risk of adverse obstetric outcomes in dichorionic twin pregnancies: a systematic review and meta-analysis. Fertil Steril. 2016;105(5):1180-1192. https://doi.org/10.1016/j.fertn stert.2015.12.131

5. Qin J, Liu X, Sheng X, Wang H, Gao S. Assisted reproductive technology and the risk of pregnancy-related complications and adverse pregnancy outcomes in singleton pregnancies: a meta-analysis of cohort studies. Fertil Steril. 2016;105(1):73-85.e6. https:// doi.org/10.1016/j.fertnstert.2015.09.007

6. Raatikainen K, Kuivasaari-Pirinen P, Hippeläinen M, Heinonen S. Comparison of the pregnancy outcomes of subfertile women after infertility treatment and in naturally conceived pregnancies. Hum Reprod. 2012;27(4):1162-1169. https://doi.org/10.1093/humrep/ des015

7. Castelli C, Perrin J, Thirion X, Comte F, Gamerre M, Courbiere B. Maternal factors influencing the decision to breastfeed newborns conceived with IVF. Breastfeed Med. 2015;10(1):26-30. https:// doi.org/10.1089/bfm.2014.0078 
8. Maheshwari A, Raja EA, Bhattacharya S. Obstetric and perinatal outcomes after either fresh or thawed frozen embryo transfer: an analysis of 112,432 singleton pregnancies recorded in the Human Fertilisation and Embryology Authority anonymized dataset. Fertil Steril. 2016;106(7):1703-1708. https://doi.org/10.1016/j.fertn stert.2016.08.047

9. Maheshwari A, Pandey S, Amalraj Raja E, Shetty A, Hamilton M, Bhattacharya $\mathrm{S}$. Is frozen embryo transfer better for mothers and babies? Can cumulative meta-analysis provide a definitive answer? Hum Reprod Update. 2018;24(1):35-58. https://doi.org/10.1093/ humupd/dmx031

10. Pinborg A, Wennerholm UB, Romundstad LB, et al. Why do singletons conceived after assisted reproduction technology have adverse perinatal outcome? Systematic review and meta-analysis. Hum Reprod Update. 2013;19(2):87-104. https://doi.org/10.1093/ humupd/dms044

11. Pandey S, Shetty A, Hamilton M, Bhattacharya S, Maheshwari A. Obstetric and perinatal outcomes in singleton pregnancies resulting from IVF/ICSI: a systematic review and meta-analysis. Hum Reprod Update. 2012;18(5):485-503. https://doi.org/10.1093/ humupd/dms018

12. Marconi N, Raja EA, Bhattacharya S, Maheshwari A. Perinatal outcomes in singleton live births after fresh blastocyst-stage embryo transfer: a retrospective analysis of 67147 IVF/ICSI cycles. Hum Reprod. 2019;34(9):1716-1725. https://doi.org/10.1093/ humrep/dez133

13. Les Enquêtes Nationales Périnatales. EPOPé. Accessed March 23, 2020. http://www.xn-epop-inserm-ebb.fr/grandes-enquetes/ enquetes-nationales-perinatales

14. Kulkarni AD, Jamieson DJ, Jones HWJ, et al. Fertility treatments and multiple births in the United States. New Engl J Med. 2013;369(23):2218-2225. https://doi.org/10.1056/NEJMo a1301467

15. Mazhar SB, Peerzada A, Mahmud G. Maternal and perinatal complications in multiple versus singleton pregnancies: a prospective two years study. J Pak Med Assoc. 2002;52(4):143-147.

16. Cutting R. Single embryo transfer for all. Best Pract Res Clin Obst Gynaecol. 2018;53:30-37. https://doi.org/10.1016/j.bpobg yn.2018.07.001

17. Palomba S, Homburg R, Santagni S, La Sala GB, Orvieto R. Risk of adverse pregnancy and perinatal outcomes after high technology infertility treatment: a comprehensive systematic review. Reprod Biol Endocrinol. 2016;14(1):76. https://doi.org/10.1186/s1295 8-016-0211-8

18. Pessione F, De Mouzon J, Deveaux A, et al. [Adverse obstetric and perinatal outcome with in vitro fertilization technology: A French nationwide population-based study]. Gynecol Obstet Fertil Senol. 2020;48(4):351-358. https://doi.org/10.1016/j.gofs.2020.02.002

19. Liu K, Case A, Cheung AP, et al. REPRODUCTIVE ENDOCRINOLOGY AND INFERTILITY COMMITTEE. Advanced reproductive age and fertility. J Obstet Gynaecol
Can. 2011;33(11):1165-1175. https://doi.org/10.1016/S1701 $-2163(16) 35087-3$

20. Okun N, Sierra S, Douglas Wilson R, et al. Pregnancy outcomes after assisted human reproduction. J Obstet Gynaecol Can. 2014;36(1):6483. https://doi.org/10.1016/S1701-2163(15)30685-X

21. Lean SC, Derricott H, Jones RL, Heazell AEP. Advanced maternal age and adverse pregnancy outcomes: A systematic review and meta-analysis. PLoS One. 2017;12(10):e0186287. https://doi. org/10.1371/journal.pone.0186287

22. Waylen AL, Metwally M, Jones GL, Wilkinson AJ, Ledger WL. Effects of cigarette smoking upon clinical outcomes of assisted reproduction: a meta-analysis. Hum Reprod Update. 2009;15(1):3144. https://doi.org/10.1093/humupd/dmn046

23. Tong VT, Kissin DM, Bernson D, et al. Maternal smoking among women with and without use of assisted reproductive technologies. J Womens Health (Larchmt). 2016;25(10):1066-1072. https://doi. org/10.1089/jwh.2015.5662

24. Kumbak B, Oral E, Bukulmez O. Female obesity and assisted reproductive technologies. Semin Reprod Med. 2012;30(6):507-516. https://doi.org/10.1055/s-0032-1328879

25. Falconer H. Pregnancy outcomes in women with endometriosis. Semin Reprod Med. 2013;31(2):178-182. https://doi. org/10.1055/s-0032-1333484

26. Vigano P, Corti L, Berlanda N. Beyond infertility: obstetrical and postpartum complications associated with endometriosis and adenomyosis. Fertil Steril. 2015;104(4):802-812. https://doi. org/10.1016/j.fertnstert.2015.08.030

27. Medical Advisory Secretariat. In vitro fertilization and multiple pregnancies: an evidence-based analysis. Ont Health Technol Assess Ser. 2006;6(18):1-63.

28. Mourad S, Brown J, Farquhar C. Interventions for the prevention of OHSS in ART cycles: an overview of Cochrane reviews. Cochrane Database Syst Rev. 2017;2017(1): https://doi.org/10.1002/14651 858.CD012103.pub2

29. Jaques AM, Amor DJ, Baker HWG, et al. Adverse obstetric and perinatal outcomes in subfertile women conceiving without assisted reproductive technologies. Fertil Steril. 2010;94(7):26742679. https://doi.org/10.1016/j.fertnstert.2010.02.043

30. Sha T, Wang X, Cheng W, Yan Y. A meta-analysis of pregnancy-related outcomes and complications in women with polycystic ovary syndrome undergoing IVF. Reprod Biomed Online. 2019;39(2):281-293. https://doi.org/10.1016/j.rbmo.2019.03.203 